eingeliefert werden mussten, konnten die Studienärzte eine genauere klinische und labordiagnostische Untersuchung veranlassen. Bei $84 \%$ diagnostizierten sie ein GBS, bei $12 \%$ eine Enzephalitis, die übrigen waren an einer Myelitis oder Optikusneuritis erkrankt. Etwa ein Drittel der Patienten hatte noch Zika-Viren oder Antikörper gegen Zika im Blut oder Urin. Vor allem im Urin ließ sich das Virus nachweisen - dies war bei $70 \%$ mit positivem PCR-Befund der Fall. Muñoz wies darauf hin, dass das ZikaVirus relativ schlecht direkt aufzuspüren ist, da es rasch nach der Infektion eliminiert wird.

\section{Komplikationen eine Woche nach der Infektion}

Ein direkter (PCR) oder indirekter Virusnachweis (Antikörper) gelang den- noch bei einem Drittel der GBS-Patienten, knapp der Hälfte der Enzephalitiskranken und bei drei von fünf Patienten mit Myelitis oder Optikusneuritis. Die Experten gehen davon aus, dass diese Krankheiten überwiegend durch das Zika-Virus verursacht wurden. Dafür spricht auch der zeitliche Zusammenhang: So traten die Nicht-GBS-Komplikationen ebenfalls in den ersten drei Monaten von 2016 gehäuft auf.

Ähnlich wie in Polynesien entwickelte sich das GBS im Median sechs Tage nach den Infektionssymptomen, die Enzephalitis fünf Tage danach, die Myelitis benötigte mit neun Tagen etwas länger.

Bei der Klinikaufnahme hatten die GBS-Patienten zu $70 \%$ eine akute demyelinisierende Polyneuropathie mit schwersten funktionellen Einschränkungen (mRS 4-5), vier Wochen später waren die Beschwerden bei etwa $40 \%$ um 1-2 mRS-Punkte zurückgegangen, $10 \%$ ging es noch schlechter, die übrigen blieben stabil. Todesfälle wurden kaum beobachtet.

An den Zika-Folgen stirbt jedoch ein beachtlicher Teil der Kinder, die mit Mikrozephalie zur Welt kommen. Darauf deutet eine brasilianische Analyse, die ebenfalls auf der Tagung vorgestellt worden ist. Von 138 Kindern, die im Nordosten des Landes mit Mikrozephalie und nachgewiesener Zika-Virus-Infektion geboren wurden, starb ein Viertel kurz darauf an den neurologischen Schäden. Thomas Müller, Springer Medizin

Session S40 Infectious Disease: The Neurology of Zika, Chikungunya, and Acute Flaccid Myelitis. 69. Annual Meeting of the American Academy of Neurology (AAN), 22. - 28.4.2017, Boston

\title{
Antikörper gegen Tau besteht ersten Sicherheitstest
}

\begin{abstract}
Ein neuer therapeutischer Antikörper gegen Tau-Protein wird von den Patienten offenbar relativ gut vertragen. Eine erste Pilotstudie offenbarte keine relevanten Sicherheitsrisiken.
\end{abstract}

$M_{i}$ it Antikörpern gegen Beta-Amyloid hatten Forscher bislang wenig Glück: Trotz vielversprechender PhaseII-Daten sind bislang alle Wirkstoffe in Phase-III-Studien gescheitert. Dies liegt vermutlich auch daran, dass Beta-Amyloid bei Demenzkranken für den Krankheitsverlauf nur noch wenig relevant ist. Hier scheinen Neurodegenerationsmarker wie Tau-Protein offenbar weitaus wichtiger zu sein. Um bereits Erkrankten zu helfen, wären Anti-Tau-Antikörper vielleicht die bessere Wahl. Inzwischen werden einige Antikörper gegen Tau-Protein entwickelt. Getestet wurde auch schon eine Tau-Vakzine (AADvac1) in Phase I bei Alzheimer-Kranken. Die Patienten haben den Impfstoff recht gut vertragen.

Ähnliches scheint nach ersten Resultaten auch für einen monoklonalen Antikörper gegen Tau-Protein zu gelten. Auf der Jahrestagung der US-Neurologengesellschaft AAN in Boston wurden
Ergebnisse einer ersten Studie mit dem Antikörper ABBV-8E12 vorgestellt. Die 30 Teilnehmer der Phase-I-Untersuchung waren alle an einer progressiven supranukleären Blickparese (PSP) erkrankt. Dabei kommt es zu massiven Tau-Ablagerungen.

Alle Patienten hatten seit mindestens fünf Jahren Krankheitssymptome, waren aber noch in der Lage, fünf Schritte ohne fremde Hilfe zu gehen. Darauf hat Dr. Hana Florian vom Unternehmen Abbvie auf der Tagung hingewiesen. 23 Patienten bekamen eine einzelne Infusion mit unterschiedlichen Dosierungen des Antikörpers (2,5 - 50 mg kg Körpergewicht), sieben Placebo.

\section{Phase-II-Studie mit 400}

Alzheimer-Patienten geplant

Unerwünschte Wirkungen traten mit dem Antikörper insgesamt nicht häufiger auf als unter Placebo, auch bildeten die Patienten keine neutralisierenden
Antikörper gegen das Medikament. In den höchsten Dosierungen wurden bei zwei Patienten ernsthafte unerwünschte Effekte beobachtet, die möglicherweise durch die Therapie bedingt waren. So kam es bei einem Patienten zu einem subduralen Hämatom aufgrund von Stürzen. Dieser Patient war aber auch schon in der Vergangenheit häufig gestürzt. Bei einem anderen Teilnehmer führten starke Agitation und Angstzustände zum Therapieabbruch; auch dieser Patient litt zuvor schon häufiger unter solchen Beschwerden. Ob der Antikörper die Symptome verursacht oder verstärkt hat, ist also fraglich.

Die Forscher um Florian gehen nach diesen Daten von einem ,akzeptablen Sicherheitsprofil“" aus und prüfen den Antikörper bereits in einer Phase-II-Studie bei 180 Patienten mit PSP. Geplant ist auch eine Studie mit 400 Patienten in einem frühen Alzheimer-Stadium. Die Betroffenen sollen jeweils vier Infusionen im Abstand von drei Monaten erhalten. In der PSP-Studie werden zwei unterschiedliche, in der Alzheimer-Studie drei verschiedene Dosierungen getestet. Thomas Müller, Springer Medizin

Emerging Science Platform Session. 69. Annual Meeting of the American Academy of Neurology (AAN). Boston, 22. - 28.4.2017, Boston 